\title{
NUTRITIONAL REQUIREMENTS AND RESPIRATORY PATTERN OF PERTUSSIS-PARAPERTUSSIS- BRONCHISEPTICUS GROUP OF MICROORGANISMS
}

\author{
HIDEO FUKUMI, EIKo SAYAMA, JUN-ICHI TOMIZAWA \\ AND TATSUJI UCHIDA \\ National Institute of Health, Tokyo, Japan
}

(Received: December 12th, 1952)

Eldering and Kendrick(1) already pointed out that Haemophilus pertussis, B. parapertussis and Brucella bronchiseptica are closely related to each other in serological and some other respects and therefore the pertussis bacillus is a misfit in the genus Haemophilus, and suggested that these three species could suitably be included in the same genus. Along this line, we studied the antigenic structures of three species of organisms by means of agglutination and absorption reaction and a part of the experimental results were already reported(2), (3). A same kind of study was reported by Andersen(4), who analyzed the K- and 0 -antigens of these organisms.

In this paper, the nutritional requirements and the respiratory patterns of these three species of organisms are submitted to a comparative study and their taxonomical significances are discussed. Studies were already made of synthetic or semisynthetic media of $\mathrm{H}$. pertussis by Hornibrook ${ }^{(5)}$ followed by Cohen, and Wheeler(6), Verwey and Sage(7), and Verwey, Thiele, Sage and Schuchardt(8). Amino acids requirements in synthetic media were studied by Kuwabara(9) (10), Imamura(11) and Arai(12). As for the terminal respiratory pattern of $\mathrm{H}$. pertussis, Abe(19) (20) (21) has published papers evidencing the presence of the tricarboxylic acid cycle pathway in this organism.

\section{NUTRITIONAL REQUIREMENTS OF PERTUSSIS-PARAPERTUSSIS-BRON- CHISEPTICUS GROUP OF ORGANISMS}

The strains used in the experiments were as follows:

Bronchisepticus :

22067: forwarded from Dr. G. Elderling.

R63: isolated from an albino rat.

Parapertussis :

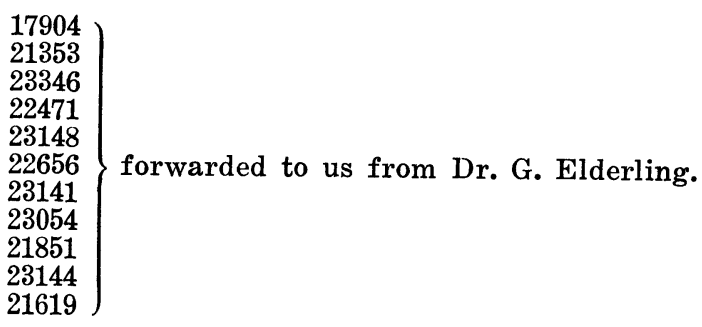




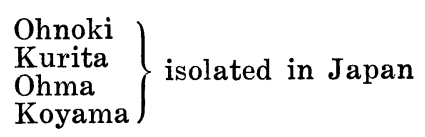

Pertussis:

Akabane: received from kitasato Institute for infectious Diseases

22490

$21763\}$ forwarded to us from Dr. P. L. Kendrick 18334

As Pertussis-Parapertussis-Bronchisepticus group of organisms is unable to utilize glucose, pyruvic acid was used as carbon source if amino acid- or sulfurrequirements were to be investigated for these organisms. The basal medium employed in the present investigation was as follows:

Pyruvic acid
$\mathrm{NaCl}$
$\mathrm{MgSO}_{4}$
$\mathrm{NH}_{4} \mathrm{H}_{2} \mathrm{PO}_{4}$
$\mathrm{~K}_{2} \mathrm{HPO}_{4}$
Distilled water

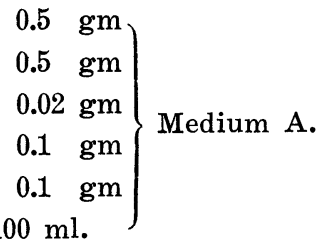

B. bronchisepticum is not able to grow in the medium A, unless this medium is supplemented with nicotinic acid, the fact which indicates that this organism requires nicotinc acid as a growth factor. This nicotinic acid requirement of this organism is, as mentioned below, a common characteristic of the PertussisParapertussis-Bronchisepticus group of organisms.

From the above mentioned fact, B. bronchisepticum grows readily with ammonium ion as nitrogen source and with sulfate ion as sulfur source if supplied with pyruvic acid and nicotinic acid as carbon source and growth factor respectively. Therefore, this organism is quite non-exact for nitrogen- and sulfur source. In this respect, the strains used in the present study as representatives of B. bronchisepticum behaved quite identically.

B. parapertussis is not able to grow in the medium A even if supplemented with nicotinic acid. This is due to the fact that this organism is exacter in respect of nitrogen and sulfur source than B. bronchisepticum. B. parapertussis is not able to use sulfate ion $\left(\mathrm{SO}_{4}{ }^{--}\right)$as sulfur source, and requires $\mathrm{SO}_{3}{ }^{--}$, $\mathrm{S}_{2} \mathrm{O}_{3}{ }^{--}, \mathrm{S}^{--}$, or cysteine, as sulfur source. In the metabolic pathway of sulfur

$$
\begin{aligned}
& \mathrm{SO}_{4}^{--} \stackrel{\mathrm{A}}{\rightarrow} \mathrm{SO}_{3}^{--} \stackrel{\mathrm{B}}{\longrightarrow} \mathrm{S}_{2} \mathrm{O}_{3}^{--} \stackrel{\mathrm{C}}{\longrightarrow} \mathrm{S}^{--} \stackrel{\mathrm{D}}{\longrightarrow} \operatorname{cyst}(\mathrm{e}) \text { ine } \rightarrow \text { protein }
\end{aligned}
$$

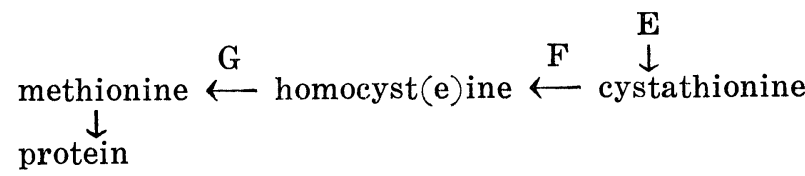

the path $\mathrm{A}$ is completely blocked in $\mathrm{B}$. bronchisepticum, but if $\mathrm{SO}_{3}{ }^{--}$is supplemented, its growth is ready. Either of $\mathrm{SO}_{3}^{--}, \mathrm{S}_{2} \mathrm{O}_{3}^{--}, \mathrm{S}^{--}$, or cyst(e)ine supports the growth of the organism, but if these sulfur sources are replaced 
with methionine, the growth is very retarded. This retardation of growth is, however, gradually repaired through serial passages in the medium containing only methionine as sulfur compound. It seems likely to us that B. parapertussis is usually splitting off an individual organism which is able to utilize methionine to synthesize cysteine or, other words, to operate the pathways G, E, and F reversibly. These individuals are therefore able to grow with either cysteine or methionine as a sole sulfur source, but on the other hand in the most individuals of $\mathrm{B}$. parapertussis, the capacity to convert methionine to cysteine is missing.

All strains of $B$. parapertussis used by us in the present study require nicotinic acid as growth factor, therefore, it should be considered that the nicotinic acid requirement is a general characteristic for this organism.

Even if the medium $\mathrm{A}$ is supplemented with nicotinic acid and an adequate sulfur source, for instance $\mathrm{Na}_{2} \mathrm{~S}_{2} \mathrm{O}_{3}$, the growth of the strains of $\mathrm{B}$. parapertussis is not so ready, and most of the strains grow after a quite long lag period. Only the strain 17904 grows comparatively readily. This long lag period of B. parapertussis in the medium devoid of amino nitrogen, is, however, gradually shortened through serial transplantation in the same medium, and that is due to the capacity obtained by the organism to utilize ammonia nitrogen as a sole nitrogen source. Furthermore, the long lag period is canceled out if the medium is supplemented with glutamic acid.

The amino acid requirement of $B$. parapertussis is best fulfilled by glutamic acid, but this is not the only amino acid for the organism but some others are able to replace it, for example aspartic acid, arginine, proline or alanine, and in lesser degree, glycine, but leucine and valine are almost of no effect.

It seems to us that glutamic acid is the most fundamental amino acid for the Pertussis-Parapertussis-Bronchisepticus group of organisms, because, as mentioned below, there is a strain, whose essential amino acid is glutamic acid, and other amino acids tested did not support its growth, even aspartic acid, arginine, or alanine. In this respect, it might be considered that those amino acid capable of replacing glutamic acid for the growth of $\mathrm{B}$. parapertussis are convertible to glutamic acid in the metabolic activity of the organisms. That the transamination reactions

$$
\text { alanine }+\alpha \text {-ketoglutaric acid }
$$

$$
\rightleftarrows \text { pyruvic acid + glutamic acid }
$$

and

$$
\text { aspartic acid }+\alpha \text {-ketoglutaric acid }
$$

$$
\rightleftarrows \text { oxalacetic acid + glutamic acid }
$$

are quite active in B. parapertussis is evidenced in the experiments, in which a mixture of $a$-ketoglutaric acid and alanine or aspartic acid is added with a suspension of $\mathrm{B}$. parapertussis, incubated at $37^{\circ} \mathrm{C}$ for hours anaerobically, and the resultant suspension is analysed by means of paper-chromatography after cen- 
trifugation in order to determine whether glutamic acid is produced or not. As for arginine, proline, or glycine the following metabolic pathway(13) of amino acid synthesis might operate in reversible way.

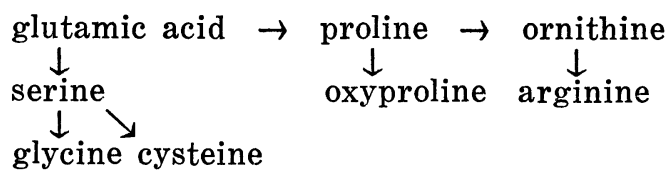

H. pertussis also requires nicotinic acid for growth as B. parapertussis does. However, the organism is very difficult to be grown in a synthetic medium. It is in general necessary to add charcoal to obtain growth of the organism in a synthetic medium. Though the strain Akabane is able to grow in ordinary broth without blood and potato extract, it does not grow in the following medium unless charcoal is supplemented:

$\begin{array}{lcc}\text { pyruvic acid } & 0.5 & \mathrm{gm} \\ \text { glutamic acid } & 0.01 & \mathrm{gm} \\ \mathrm{Na}_{2} \mathrm{~S}_{2} \mathrm{O}_{3} & 0.01 \mathrm{gm} \\ \text { nicotinic acid } & 10^{-4} & \mathrm{M} \\ \mathrm{NaCl} & 0.5 & \mathrm{gm} \\ \mathrm{MgSO}_{4} & 0.02 & \mathrm{gm} \\ \mathrm{NH}_{4} \mathrm{H}_{2} \mathrm{PO}_{4} & 0.1 & \mathrm{gm} \\ \mathrm{K}_{2} \mathrm{HPO}_{4} & 0.1 & \mathrm{gm} \\ \text { distilled water } & 100 \mathrm{ml}\end{array}$

If the medium $B$ is added with charcoal at a concentration of $0.05 \%$, the strain Akabane grows and is serially transplantable. The incubation period was, in one experiment, 5 days at the first passage from broth culture, 6 days at the second, 4 days at the third, 3 days at the fourth, and 2-3 days at each passage thereafter. From this experiment it is clear that the strain Akabane grows in the medium B with charcoal $0.05 \%$. However, from the fourth subculture in the medium $B$ with charcoal, transplantation was made into the medium B itself deprived of charcoal, and this time the organism grew after 5 days' incubation. Serial transplantation was again made from this culture and the incubation period for growth was at first about 5 days, but after 5 passages without charcoal, it became two days and thereafter remained constant, about 30-48 hours. Thus the strain Akabane was made to grow readily and the study of nutritional requirements of this organism was carried out by using this trained culture.

The strain Akabane is not able to grow with $\mathrm{SO}_{4}^{--}$as sulfur source, but $\mathrm{S}_{2} \mathrm{O}_{3}^{--}$or cyst(e)ine supports its growth fully. This characteristic is the same as that of B. parapertussis as for sulfur source. Furthermore, methionine also does not support the growth of the organism as a sole sulfur source, namely, if $\mathrm{Na}_{2} \mathrm{~S}_{2} \mathrm{O}_{3}$ in the medium $\mathrm{B}$ was replaced with methionine, it is unable to support its growth. In this respect the strain Akabane is more exact than 
B. parapertussis which is able to give rise to such a mutant that grows with methionine as sulfur source.

The strain Akabane requires, of course, nicotinic acid as a growth factor. If nicotinic acid is omitted from the medium $B$, it does not give growth to the organism.

The amino acid requirements of the strain Akabane are of interest in comparison of those of the other pertussis strains described later. As mentioned above, glutamic acid is essential for the Akabane strain in the medium B and, therefore, the medium $B$ does not support the growth of this strain if this amino acid is omitted. Further, this amino acid is unable to be replaced with asparatic acid, arginine or alanine. This is the characteristic of the Akabane which differentiate it from $B$. parapertussis.

At last, the nutritional requirements of the typical $H$. pertussis strains, 22490, 21763 and 18334 are to be described. These organisms were at first unable to grow in the medium B supplemented with charcoal $0.05 \%$, however, we could obtain the growth of these organisms in the following medium, which were able to be transplanted serially:

$\left.\begin{array}{lcc}\text { pyruvic acid } & 0.5 & \mathrm{gm} \\ \text { glutamic acid } & 0.01 & \mathrm{gm} \\ \text { aspartic acid } & 0.01 & \mathrm{gm} \\ \text { arginine } & 0.01 \mathrm{gm} \\ \text { cystine } & 0.01 & \mathrm{gm} \\ \text { nicotinic acid } & 10^{-4} & \mathrm{M} \\ \mathrm{NaCl} & 0.5 & \mathrm{gm} \\ \mathrm{MgSO}_{4} & 0.02 \mathrm{gm} \\ \mathrm{NH}_{4} \mathrm{H}_{2} \mathrm{PO}_{4} & 0.1 & \mathrm{gm} \\ \mathrm{K}_{2} \mathrm{HPO}_{4} & 0.1 & \mathrm{gm} \\ \text { charcoal } & 0.05 \mathrm{gm} \\ \text { distilled water } & 100 \mathrm{ml}\end{array}\right\}$

Nicotinic acid is, of course, an essential growth factor for these pertussis strains also.

At first, these pertussis organisms grow in the medium $\mathrm{C}$, about 10 days after inoculation. This rather longer incubation period is maintained for the first 5 or 10 passages and then gradually or fairly rapidly shortened to 2-3 days (Table 1). The nutritional requirements of these pertussis strains were investigated either during the course of passages for adaptation or after these organisms became to grow with 2-3 days, incubation period. It is the same as before that, when we say "be able to grow", it means the growth which is transplantable serially.

In the first place, their amino acid requirements are to be mentioned. Same as in the case of the strain Akabane, glutamic acid seems to play an essential 
Table 1. Incubation period for growth of the strains of $\mathrm{H}$. pertussis during serial passages

\begin{tabular}{c|c|c|c}
\hline \multirow{2}{*}{ Passage number } & \multicolumn{3}{|c}{ Strain } \\
\cline { 2 - 4 } & 21763 & 22490 & 18334 \\
\hline 1 & 5 (days) & 4 (days) & 4 (days) \\
2 & 12 & 10 & 14 \\
3 & 10 & 5 & 8 \\
4 & 9 & 6 & 12 \\
5 & 5 & 5 & 9 \\
6 & 5 & 5 & 3 \\
7 & 10 & 8 & 2 \\
8 & 4 & 4 & 3 \\
9 & 3 & 5 & 2 \\
10 & 3 & 2 & 2 \\
11 & 5 & 2 & 2 \\
12 & 4 & 2 & \\
13 & 3 & & \\
14 & 2 & & \\
15 & 2 & & \\
16 & 3 & & \\
\end{tabular}

role in growth maintenance for the pertussis strains now under study. These strains were, namely, grown in the medium more or less readily which is the same as the medium $\mathrm{C}$ except that it is deprived of aspartic acid and arginine:

$\left.\begin{array}{lcc}\text { pyruvic acid } & 0.5 & \mathrm{gm} \\ \text { glutamic acid } & 0.01 \mathrm{gm} \\ \text { cystine } & 0.01 \mathrm{gm} \\ \text { nicotinic acid } & 10^{-4} & \mathrm{M} \\ \mathrm{NaCl} & 0.5 \mathrm{gm} \\ \mathrm{MgSO}_{4} & 0.02 \mathrm{gm} \\ \mathrm{NH}_{4} \mathrm{H}_{2} \mathrm{PO}_{4} & 0.1 \mathrm{gm} \\ \mathrm{K}_{2} \mathrm{HPO}_{4} & 0.1 \mathrm{gm} \\ \text { charcoal } & 0.05 \mathrm{gm} \\ \text { distilled water } & 100 \mathrm{ml}\end{array}\right\}$ Medium D.

Their growth in the medium $\mathrm{D}$ is, however, as mentioned above, a little poorer or sometimes requires a longer incubation period.

In the next experimental step, it was attempted to decide whether there is any other amino acid capable of replacing glutamic acid to support the growth of the pertussis organism in the medium $\mathrm{D}$, and proline was found to do it but arginine, alanine, glycine, citruline and histidine were unable to replace it. The medium D in which glutamic acid is replaced by proline, is able to support the growth of those pertussis organisms in question almost as well as the original medium D. In this respect, proline and glutamic acid are almost equivalent in their capacity to support their growth. 
In this point of view, the behavior of aspartic acid is of interest. This amino acid is indeed able to support the growth of the pertussis organisms in the place of glutamic acid in the medium $D$, but in the first several passages in it from the original $\mathrm{D}$ culture, the lag period is pretty long and it is gradually shortened to about 2 days, for instance follows:

$\begin{array}{ll}5 \text { days } & \text { for 1st passage } \\ 3 \text { days } & \text { for 2nd passage } \\ 2 \text { days } & \text { for 3rd passage } \\ 2 \text { days } & \text { for 4th passage } \\ 9 \text { days } & \text { for 5th passage } \\ 4 \text { days } & \text { for 6th passage } \\ 2 \text { days } & \text { for 7th passage } \\ 2 \text { days } & \text { for } 8 \text { th passage }\end{array}$

This fact seems to indicate that aspartic acid is much less active for their growth than proline or glutamic acid.

Here we have come to deal with sulfur source of the pertussis organisms in question. In this respect, the strain 21763 behaves differently from the other two, namely, cystine, but not $\mathrm{Na}_{2} \mathrm{~S}_{2} \mathrm{O}_{3}$, does support the growth of the strain 21763, while the strains 22490 and 18334 are able to grow on $\mathrm{Na}_{2} \mathrm{~S}_{2} \mathrm{O}_{3}$, if this compound replaces cystine in the medium $C$. This fact is of interest in the point of view that Imamura reports the growth of $\mathrm{H}$. pertussis on $\mathrm{Na}_{2} \mathrm{~S}_{2} \mathrm{O}_{3}$, while Yamamoto et al ${ }^{(20)}$ reported that the strains employed in their study grew on cystine but not on $\mathrm{Na}_{2} \mathrm{~S}_{2} \mathrm{O}_{3}$.

Though the strains 22490 and 18334, as mentioned above, grow on $\mathrm{Na}_{2} \mathrm{~S}_{2} \mathrm{O}_{3}$ in the medium $\mathrm{C}$, in which cystine is replaced with $\mathrm{Na}_{2} \mathrm{~S}_{2} \mathrm{O}_{3}$, they are not so ready to grow on $\mathrm{Na}_{2} \mathrm{~S}_{2} \mathrm{O}_{3}$ in the medium $\mathrm{D}$, where cystine is replaced by $\mathrm{Na}_{2} \mathrm{~S}_{2} \mathrm{O}_{3}$. This may indicate that the utilization of $\mathrm{Na}_{2} \mathrm{~S}_{2} \mathrm{O}_{3}$ as sulfur source by these strains are not so smooth unless amino acids like aspartic acid or arginine are supplied in addition to glutamic acid.

\section{TERMINAL RESPIRATORY PATTERN OF PERTUSSIS-PARAPERTUSSIS- BRONCHISEPTICUS-GROUP OF ORGANISMS}

Though Abe reported that in $\mathrm{H}$. pertussis the tricarboxylic acid cycle is operating, conclusive evidence seems to be still lacking. That each member of the Pertussis-Parapertussis-Bronchisepticus-group of organisms is able to oxidize the members of the tricarboxylic acid cycle is evidenced through oxygen consumption experiments by means of Warburg manometeric apparatus as shown in Fig. 1, 2, and 3. Pyruvic acid, acetic acid, citric acid, $\alpha$-ketoglutaric acid, succinic acid, fumaric acid and malic are readily oxidized by each member of the group of organisms.

These experimental results alone are not conclusively evidencing for the tricarboxylic acid cycle operation in the group of organisms, and we carried out further experiments as follows: 


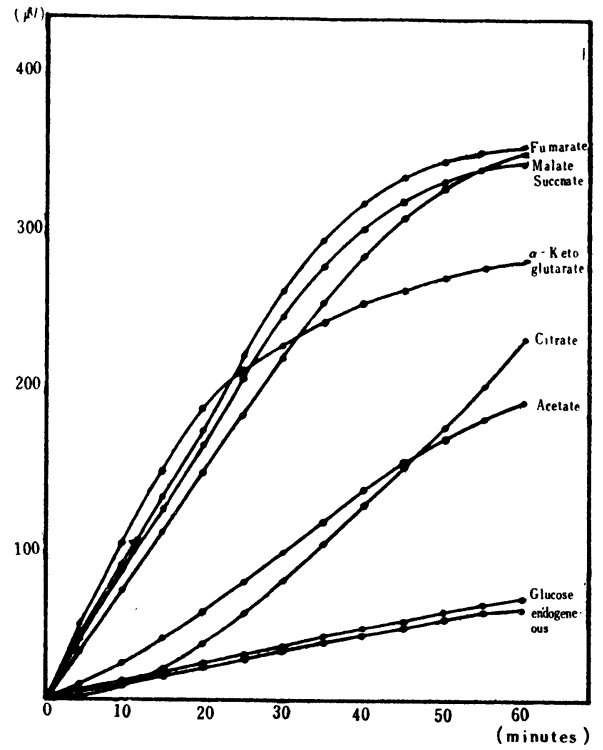

Fig. 1. Oxygen consumption of $\mathrm{H}$. pertussis

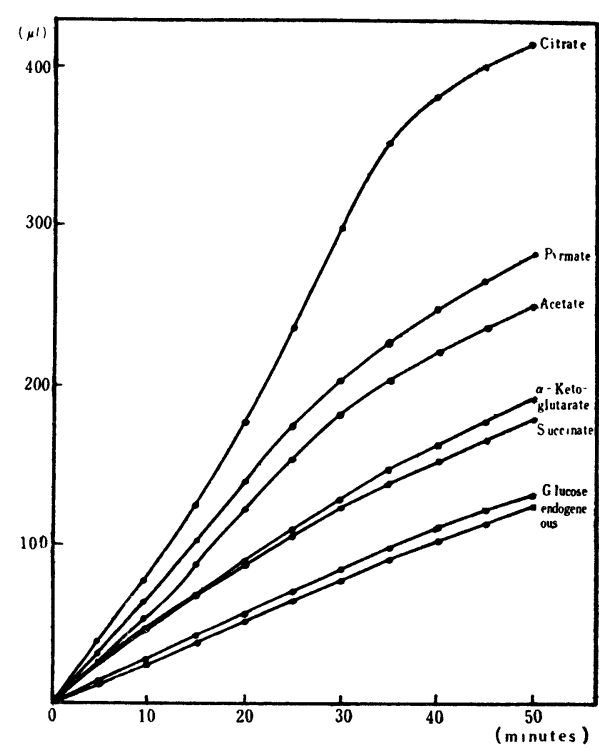

Fig. 2. Oxygen consumption of B. parapertussis

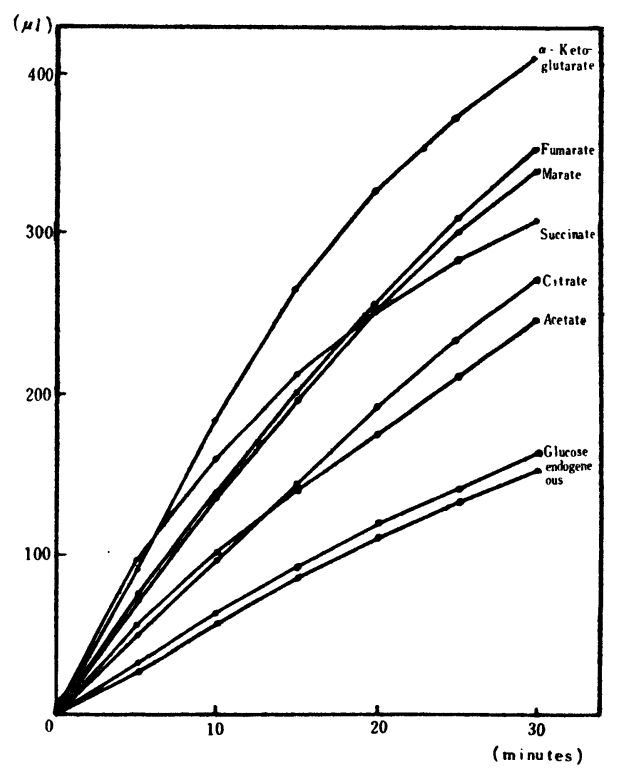

Fig. 3. Oxygen consumption of $\mathrm{B}$. bronchisepticum

\section{a. Existence of aconitase}

In this series of experiments whole bacterial cells were employed. They are suspended in phosphate buffer of $\mathrm{pH} 7.4$, which contains isocitric acid at a final concentration of $5 \times 10^{-3} \mathrm{M}$. and incubated at $25^{\circ} \mathrm{C}$. Citric acid is measured through the method reported by Natelson, Pincus, and Lugovog(14) from time to 
time. The result is shown in Table 2, which clearly indicates the existence of aconitase in the group of organisms.

Table 2. Aconitase activity of Pertussis-ParapertussisBronchisepticus group

\begin{tabular}{l|c|c|c|c|c}
\hline \multirow{2}{*}{ Organisms } & \multicolumn{5}{|c}{ Time } \\
\cline { 2 - 6 } & $15^{\prime}$ & $30^{\prime}$ & 1 hours & 2 hours & 20 hours \\
\hline B. bronchisepticum & $(\mu \mathrm{M}) \#$ & $(\mu \mathrm{M})$ & $(\mu \mathrm{M})$ & $(\mu \mathrm{M})$ & $(\mu \mathrm{M})$ \\
B. parapertussis & $/$ & 0.4 & $/$ & $/$ & $>2$ \\
H. pertussis & $/$ & 0.05 & 0.2 & $/$ & 4 \\
\# amount of citric acid formed & 0.02 & 0.04 & $/$ & 0.2 & $/$
\end{tabular}

b. Production of $\alpha$-ketoglutaric acid from citric acid

As in this series of experiments the transamination reaction

L-glutamic acid+oxalacetic acid $\rightleftarrows \alpha$-ketoglutaric acid+L-aspartic acid was utilized, it was first necessary to confirm the existence of this transaminase in the organisms in question. In this respect, the first step of the experiments was carried out to know whether the transaminating enzymes conducting the above reaction or the below:

L-glutamic acid+pyruvic acid $\rightleftarrows \alpha$-ketoglutaric acid $+\mathrm{L}$-alanine do exist, according to the method described by Housewright and Thorne ${ }^{(15)}$ in B. anthracis.

After the existence of these two enzymes was confirmed, the next step was carried out according to Altenbern and Housewright(16), to know if the reaction

$$
\text { citric acid } \longrightarrow \alpha \text {-ketoglutaric acid }
$$

proceeds. The cell suspension was incubated with citric acid for 18-24 hours under aerobic condition in addition of arsenite ion to prevent further oxidation of $\alpha$-ketoglutaric acid formed.

The experimental result clearly demonstrates that $\alpha$-ketoglutaric acid is formed from citric acid in the members of the Pertussis-Parapertussis-Bronchisepticus group.

From the above mentioned experiments, it is quite suggesting that the tricarboxylic acid cycle is operating in the organisms of the Pertussis-Parapertussis-Bronchisepticus group.

\section{CYTOCHROM SYSTEM IN THE ORGANISMS OF PERTUSSIS-PARA- PERTUSSIS-BRONCHISEPTICUS GROUP}

The cytochrom systems of several microorganisms were already investigated and reported by Yaoi and Tamiya(17), or Fujita and Kodama(18), but as for those of the Pertussis-Parapertussis-Bronchisepticus group of organisms, there has been any paper published as far as the present authors know. As mentioned above, a member of the Pertussis-Parapertussis-Bronchisepticus group, namely, Bronchisepticus is classified as Brucella by certain authors, while the other members especially $\mathrm{H}$. pertussis find their place in the genus Haemophilus. According to our investigation, $H$. influenzae, the representative of the genus Haemophilus 
and Brucella abortus are different from each other in respect of their cytochrom systems. Therefore, it seems to be very interesting to compare the cytochrom systems of Pertussis-Parapertussis-Bronchisepticus group to that of Haemophilus or of Brucella.

The strains employed are as follows:

H. influenzae Shiga*

H. influenzae Kibuse*

H. influenzae Ihata*

Brucella:

B. abortus $125^{* *}$

Pertussis-Parapertussus-Bronchisepticus group

H. pertussis 22490

H. parapertussis 17903

B. bronehisepticus 22067

Grown organisms on agar medium were collected with physiological saline and then three times washed with centrifugations and resuspensions. The bacterial paste obtained were ground with glass powder, extracted with $\mathrm{M} / 50$ phosphate buffer of $\mathrm{pH} 7.4$ and the supernatant was centrifuged at 12,000 RPM for 20 minutes. The resultant supernatant was submitted to spectrophotometric analysis by means of Beckman Model DU spectrophotometer, after aeration for oxidized and addition of sodium hydrosulphite for reduced cytochroms.

The results obtained were summarized in the Figures 4-9. In the figures, the ordinate expresses the optical density and the abscissa the wave length in $\mathrm{m} \mu$. The abscissa is graduated inversely proportionally to $\lambda^{4}$, in order to make the analysis of absorption spectra of the cytochroms themselves easy because the intensity of non-specific light-scattering is inversely proportional to $\lambda^{4}$ and therefore the influence of the back ground against absorption spectra of cytochroms is to express itself linearly in such a scale.

As seen in Fig. 4-6, the cytochrom systems of $H$. influenzae are different from those of $B$. abortus. In $H$. influenzae, one of the peaks of absorption spectra lies on the wave length of $552 \mathrm{~m} \mu$, while, in B. abortus, no peak exists on the wave length of $522 \mathrm{~m} \mu$, but on that of $560 \mathrm{~m} \mu$. In other words, $\mathrm{H}$. influenzae possesses so-called cytochrom $\mathrm{c}$ but B. abortus has so-called cytochrom b. There is a discrepancy between our experimental results and those of Yaoi and Tamiya(17), or Fujita and Kodama(18), which report that $H$. influenzae possesses cytochrom b, but not c. Taking this discrepancy into consideration, experiments were repeated for analysis of cytochrom system of this microorganisms by using three different strains, but the result was the same every time, as before.

As for the cytochrom systems of the Pertussis-Parapertussis-Bronchisepticus group of organisms, Fig. 7-9 show that they are quite similar in the point that the main peak of absorption spectra lies on the wavelength of $552 \mathrm{~m} \mu$. namely the main component of cytochrom in them is socalled cytochrom c.

* Received from the Type Culture Collection of the Institute for Infectious Diseases. ** Received from the Japanese Government Experimental Station for Animal Hygiene. 
From the above mentioned result, it might be concluded that the PertussisParapertussis-Bronchisepticus group of organisms lies nearer to $\mathrm{H}$. influenzae rather than to Brucella abortus.
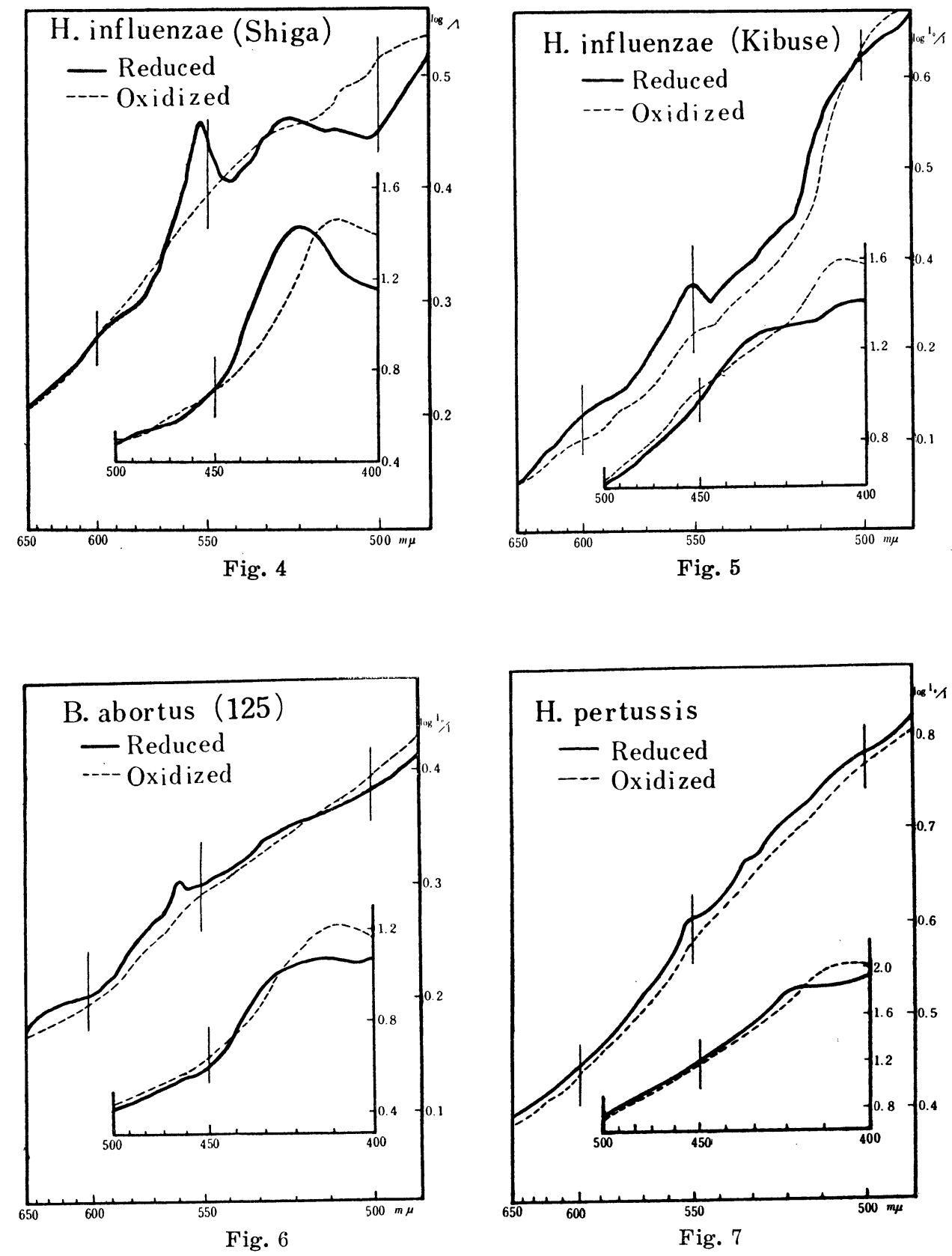


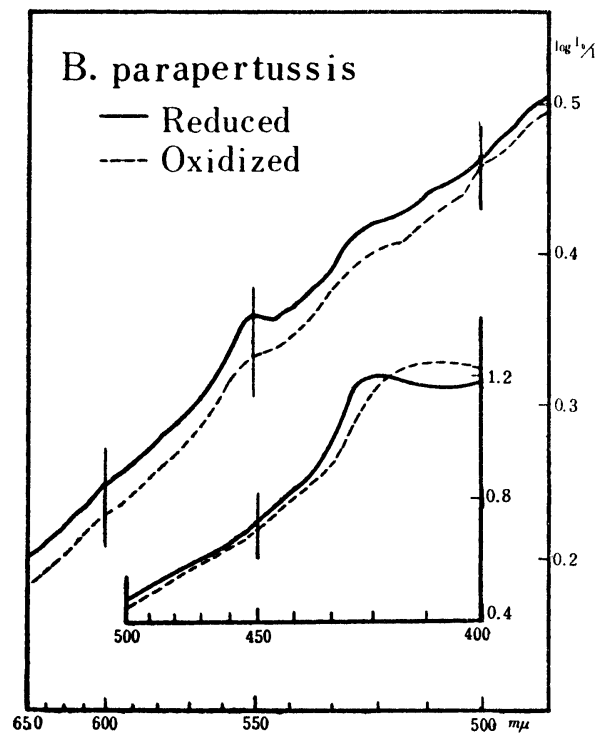

Fig. 8

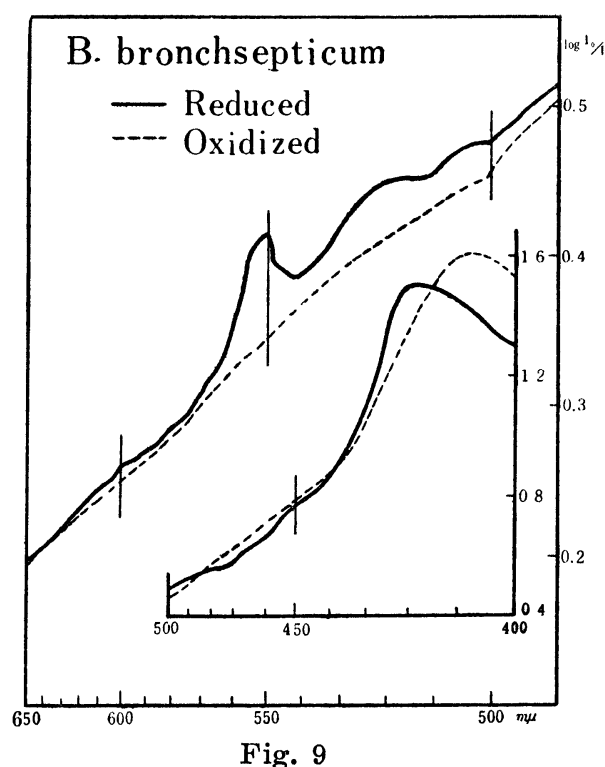

Fig. 9

\section{Discussion}

It has become considerably clear from the present investigation that the three species of microorganisms, namely, H. pertussis, B. parapertussis, and B. bronchisepticum could reasonably be classified into one group, Pertussis-Parapertussis-Bronchisepticus group, together with their remarkable characteristics that they could not ferment any of the pentoses, hexoses, or the compounds alike.

The cytochrom systems of the members of this group are identical with each other in that the main absorption peak lies on the wave length of $552 \mathrm{~m} \mu$. Another characteristic possessed in common by these organisms is that the tricarboxylic acid cycle is operating in their metabolic pathways. From this fact and the amino acid requirements of the group of organisms, the basal metabolic pathways might be suggested as in Fig. 10. In B. bronchisepticum, these pathways are all readily operating, and especially as the aminating process $\alpha$-ketoglutaric acid $\rightarrow$ glutamic acid with expense of $\mathrm{NH}_{4}{ }^{-}$is easy, so that no amino acid is required for growth.

In B. parapertussis, however, the aminating process $\alpha$-ketoglutaric acid $\rightarrow$ glutamic acid (A in Fig. 10) is rather scantily operating, so if glutamic acid is supplied, the growth of the organisms becomes very ready and rapid. The weakness of this reaction is, on the other hand, supplemented by the process of training the organisms in the medium devoid of glutamic acid. It is not clear yet whether this process is due to real training or selection of the individuals having rather more active aminating reaction of $\alpha$-ketoglutaric acid. That proline, arginine or glycine is capable to support the growth more or less in the place of glutamic acid might be attributed to the reversibilty of the pathways $B, C$, 
and $\mathrm{D}$ or $\mathrm{E}$ and $\mathrm{F}$, and the active operation of the transamination reactions from alanine or aspartic acid to form glutamic acid during the growth process might be the reason why these amino acids are able to replace glutamic acid in B. parapertussis.

The pathway A in Fig. 10 is entirely blocked in the H. pertussis, because in this organism glutamic acid is essential for growth and its absence never supports the growth. As far as examined by us, proline is the only amino acid replacing glutamic acid and this may indicate that the pathway $B$ in Fig. 10 alone is reversible during the growth process in this organism. Aspartic acid has a very poor activity to support growth of $\mathrm{H}$. pertussis in the place of glutamic acid, and this action is strengthened by training namely passage in the medium containing aspartic acid but not glutamic acid. This is considered to be due to the weakness of the aspartic- $\alpha$-ketoglutaric transamination reaction in this organism, that is indeed evidenced by the experiment of resting bacterial organisms. Alanine is not effective in this organism for replacing glutamic acid.

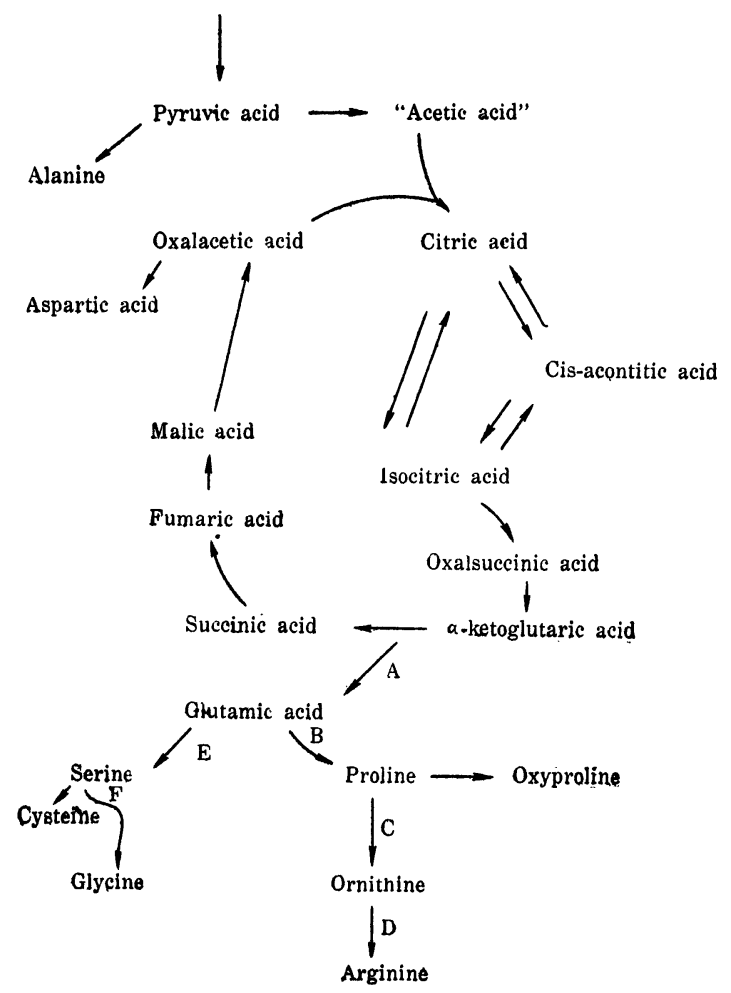

As mentioned above, there is a step-by-step correlation through a basal or fundamental metabolic pathway in the members of the Pertussis ParapertussisBronchisepticus group. This is true in S-metabolism as well as in N-metabolism through the tricarboxylic acid cycle. 


\section{SUMMARY}

The group Pertussis-Parapertussis-Bronchisepticus is proposed and the reasons are alleged from the standpoints of metabolic activities and pathways and of respiratory patterns.

\section{REFERENCES}

(1) Elderling, G., and Kendrick, P. L.: Incidence of parapertussis in the Grand Rapids ares as indicated by 16 years' experience with diagnostic cultures, Am. J. Public Health, 42, 27, 1952.

(2) Fukumi, H., and Uchida, T.: reported before the Meeting of the Committee for Pertussis Study in October, 1951.

(3) Fukumi, H., and Uchida, T.: reported before the Meeting of the Committee for Pertussis Study in June 1952.

(4) Andersen, E. K.: Serological studies on H. pertussis, H. parapertussis and H. bronchisepticus. Preliminary report, Acta Path. Microb. Scand., 30, 54-58, 1952.

(5) Hornibrook, J. W.: Cultivation of phase I H. pertussis in a semisynthetic liquid medium, Rep. Health Reports, 54, 1847-1851, 1939.

(6) Cohen, S. M., and Wheeler, M. W.: Pertussis vaccine prepared with Phase I cultures growth in fluid medium, Am. J. Publ. Health, 36, 371-376, 1946.

(7) Verwey, W. F., and Sage, D. N.: An improved liquid culture medium for the growth of hemophilus pertussis, J. Bact., 49, 520-525, 1945.

(8) Verwey, W. F., Thiele, E. H., Sage, D. N., and Schuchardt, L. F.: A simplified liquid culture medium for the growth of hemophilus pertussis, J. Bact., 58, 127-134, 1949.

(9) Kuwabara, S.: Synthetic media for several bacterial species, Saishin Igaku, 3, 337-344, 1948. (in Japanese).

(10) Kuwabara, S.: Experimental Studies of Bacterial growth factors, II. Growth of several bacterial species on synthetic media, Nisshin Igaku, 35, 519-522, 1948. (in Japanese).

(11) Imamura, P. S.: Growth requirements of Hemophilus pertussis with special reference to the production of thermolibile toxin, Jap. J. exp. Med., 22, 49-52, 1952.

(12) Arai, S.: Studies on the synthetic media of Haemophilus pertussis. I. Jap. J. Pact., 4, 343-346, 1950. (in Japanese).

(13) Hawrowitz, F.: Fortschritte der Biochemie, 1938-1947, S. 24, 1950.

(14) Natelson, S., Pincus, J. B., and Lugovog, J. K.: Microestimation of citric acid: a new colorimetric reaction for pentabromacetone, J. biol. Chem., 175, 745-750, 1948.

(15) Housewright, R. D., and Thorne, C. C.: Synthesis of glutamic acid and glutamyl polypeptide by Bacillus anthracis, J. Bact., 60, 89-100, 1950.

(16) Altenbern, R. A., and Housewright, R. D.: Alanine and carbohydrate oxidation by smooth Brucella abortus, J. Bact., 62, 97-105, 1951.

(17) Yaoi, H. and Tamiya, H.: On the respiratory pigment, cytochrome, in bacteria. Proc. Imper. Acad, 4, 436-439, 1928.

(18) Fujita, A., and Kodama, T.: Untersuchungen über Atmung und Gärung pathogener Bakterien, III. Mitteiling: Über Cytochrom und das sauerstoffübertragende Ferments, sowie die Atmungshemmung der pathogenen Bakterien durch CO und HCN. Biochem. Z., 273, 187-199, 1934. 
(19) Abe, S.: Metabolism of H. pertussis I. Dehydrogenase system. Jap. J. Bact., 7, 67-70, 1952. (in Japanese).

(20) Abe, S.: Metabolism of H. pertussis. II. metabolic mode. Jap. J. Bact., 7, 81-94, 1952. (in Japanese).

(21) Abe, S.: Metabolism of H. portussis, III. mechanism of action of carbon, red cell and soluble starch on the medium, Jap. J. Bact., $7,85-89,1952$. (in Japanese).

(22) Yamamoto, I., et al: Reports of the Committee for Pertussis Study, 1951. (in Japanese). 